\title{
On the Ulam Stability of Cauchy Functional Equation in IFN-Spaces
}

\author{
A. Alotaibi ${ }^{1}$, M. Mursaleen ${ }^{2, *}$, H. Dutta ${ }^{3}$ and S. A. Mohiuddine ${ }^{1}$ \\ ${ }^{1}$ Department of Mathematics, Faculty of Science, King Abdulaziz University, P.O. Box 80203, Jeddah 21589, Saudi Arabia \\ ${ }^{2}$ Department of Mathematics, Aligarh Muslim University, Aligarh 202002, India \\ ${ }^{3}$ Department of Mathematics, Gauhati University, Guwahati 781014, Assam, India
}

Received: 26 May. 2013, Revised: 30 Sep. 2013, Accepted: 1 Oct. 2013

Published online: 1 May. 2014

\begin{abstract}
The aim of this paper is to establish some stability results concerning the Cauchy functional equation $f(x+y)=f(x)+f(y)$ in the framework of intuitionistic fuzzy normed spaces.
\end{abstract}

Keywords: $t$-norm, $t$-conorm, intuitionistic fuzzy normed space, Cauchy functional equation, Hyers-Ulam stability

\section{Introduction and Preliminaries}

The notion of fuzzy sets was first introduced by Zadeh [40] in 1965 which is a powerful hand set for modeling uncertainty and vagueness in various problems arising in the field of science and engineering. Among various developments of this new theory, a progressive development has been made to find the fuzzy analogues of the classical set theory. In fact the fuzzy theory has become an area of active researches for the last four decades. It has a wide range of applications in the field of science and engineering, e.g. population dynamics [4], chaos control [6], computer programming [7], nonlinear dynamical systems [9], fuzzy physics [15], etc. The fuzzy topology [11] proves to be a very useful tool to deal with such situations where the use of classical theories breaks down.

Stability problem of a functional equation was first posed by Ulam [37] which was answered by Hyers [10] and then generalized by Aoki [3] and Rassias [35] for additive mappings and linear mappings, respectively. Since then several stability problems for various functional equations have been investigated in [12], [13], [14], [16], [20], [22], [23], [33], [34] and [35]. Recently, the stability problem for mixed type quadratic-additive functional equation, Jensen functional equation, additive functional equation, Pexiderized quadratic functional equation, cubic functional equation and mixed type additive cubic functional equations have been considered in [1], [17], [19], [25], [27] [30] and [38] in the intuitionistic fuzzy normed spaces. Note that the idea of intuitionistic fuzzy normed space was introduced in [36] and further studied in [24], [28] [29], [31], [32] and [39]. Quite recently, Chang [5] has established the stability of higher ring derivation in intuitionistic fuzzy Banach algebras associated to the Jensen type functional equation. In the recent past, Alotaibi and Mohiuddine [2] established the Ulam stability of a cubic functional equation in random 2-normed spaces, while the notion of random 2-normed spaces was introduced by Goleţ [8] and further studied in $[18,26,21]$.

Now, we recall some notations and basic definitions which will be used throughout the paper.

Definition 1.1. A binary operation $*:[0,1] \times[0,1] \rightarrow[0,1]$ is said to be a continuous $t$-norm if it satisfies the following conditions:

(a) $*$ is associative and commutative, $(b) *$ is continuous, (c) $a * 1=a$ for all $a \in[0,1],(d) a * b \leq c * d$ whenever $a \leq c$ and $b \leq d$ for each $a, b, c, d \in[0,1]$.

Definition 1.2. A binary operation $\diamond:[0,1] \times[0,1] \rightarrow[0,1]$ is said to be a continuous $t$-conorm if it satisfies the following conditions:

$\left(a^{\prime}\right) \diamond$ is associative and commutative, $\left(b^{\prime}\right) \diamond$ is continuous, $\quad\left(c^{\prime}\right) \quad a \diamond 0=a$ for all $a \in[0,1]$, $\left(d^{\prime}\right) a \diamond b \leq c \diamond d$ whenever $a \leq c$ and $b \leq d$ for each

\footnotetext{
${ }^{*}$ Corresponding author e-mail: mursaleenm@gmail.com
} 
$a, b, c, d \in[0,1]$.

Using the notions of continuous $t$-norm and $t$-conorm, Saadati and Park [36] introduced the concept of intuitionistic fuzzy normed space as follows:

Definition 1.3. The five-tuple $(X, \mu, v, *, \diamond)$ is said to be an intuitionistic fuzzy normed spaces (for short, IFNS) if $X$ is a vector space, $*$ is a continuous $t$-norm, $\diamond$ is a continuous $t$-conorm, and $\mu, v$ are fuzzy sets on $X \times(0, \infty)$ satisfying the following conditions for each $x, y \in X$ and $s, t>0$

(i) $\mu(x, t)+v(x, t) \leq 1$, (ii) $\mu(x, t)>0$, (iii) $\mu(x, t)=1$ if and only if $x=0$, (iv) $\mu(\alpha x, t)=\mu\left(x, \frac{t}{|\alpha|}\right)$ for each $\alpha \neq 0, \quad(v) \quad \mu(x, t) * \mu(y, s) \leq \mu(x+y, t+s)$, (vi) $\mu(x, \cdot):(0, \infty) \rightarrow[0,1]$ is continuous, (vii) $\lim _{t \rightarrow \infty} \mu(x, t)=1$ and $\lim _{t \rightarrow 0} \mu(x, t)=0$, (viii) $v(x, t)<1$, (ix) $v(x, t)=0$ if and only if $x=0$, (x) $v(\alpha x, t)=v\left(x, \frac{t}{|\alpha|}\right)$ for each $\alpha \neq 0, \quad(x i)$ $v(x, t) \diamond v(y, s) \geq v(x+y, t+s), \quad(x i i)$ $v(x, \cdot):(0, \infty) \rightarrow[0,1]$ is continuous, (xiii) $\lim _{t \rightarrow \infty} v(x, t)=0$ and $\lim _{t \rightarrow 0} v(x, t)=1$.

In this case $(\mu, v)$ is called an intuitionistic fuzzy norm.

Example 1.4. Let $(X,\|\|$.$) be a normed space, a * b=a b$ and $a \diamond b=\min \{a+b, 1\}$ for all $a, b \in[0,1]$. For all $x \in X$ and every $t>0$, consider

$\mu(x, t)=\left\{\begin{array}{ll}\frac{t}{t+\|x\|} & \text { if } t>0 \\ 0 & \text { if } t \leq 0 ;\end{array} \quad\right.$ and $\quad v(x, t)= \begin{cases}\frac{\|x\|}{t+\|x\|} & \text { if } t>0 \\ 0 & \text { if } t \leq 0\end{cases}$

Then $(X, \mu, v, *, \diamond)$ is an IFNS.

The concepts of convergence and Cauchy sequences in intuitionistic fuzzy normed space are studied in [36].

Let $(X, \mu, v, *, \diamond)$ be an IFNS. A sequence $x=\left(x_{k}\right)$ is said to be intuitionistic fuzzy convergent to $L \in X$ if, for every $\varepsilon>0$, there exists $k_{0} \in \mathbb{N}$ such that $\mu\left(x_{k}-L, t\right)>1-\varepsilon$ and $v\left(x_{k}-L, t\right)<\varepsilon$ for all $k \geq k_{0}$. In this case we write $(\mu, v)-\operatorname{limx}_{k}=L$ or $x_{k} \stackrel{(\mu, v)}{\longrightarrow} L$ as $k \rightarrow \infty$

Let $(X, \mu, v, *, \diamond)$ be an IFNS. A $x=\left(x_{k}\right)$ is said to be intuitionistic fuzzy Cauchy sequence if, for every $\varepsilon>0$ and $t>0$, there exists $k_{0} \in \mathbb{N}$ such that $\mu\left(x_{k}-x_{l}, t\right)>1-\varepsilon$ and $v\left(x_{k}-x_{l}, t\right)<\varepsilon$ for all $k, l \geq k_{0}$

An IFNS $(X, \mu, v, *, \diamond)$ is said to be complete if every intuitionistic fuzzy Cauchy sequence is intuitionistic fuzzy convergent in $(X, \mu, v, *, \diamond)$. In this case $(X, \mu, v)$ is called intuitionistic fuzzy Banach space.

\section{Main Results}

We start our work with an intuitionistic fuzzy version of the Hyers-Ulam-Rassias stability in which we uniformly approximate a 'uniform' approximate additive mapping.
Theorem 2.1. Let $X$ be a linear space and $(Y, \mu, v)$ be an intuitionistic fuzzy Banach space. Let $\varphi: X \times X \rightarrow[0, \infty)$ be a control function such that

$$
\tilde{\varphi}(x, y)=\sum_{n=0}^{\infty} 2^{-n} \varphi\left(2^{n} x, 2^{n} y\right)<\infty
$$

for all $x, y \in X$. Let $f: X \rightarrow Y$ be a uniformly approximately additive function with respect to $\varphi$ in the sense that

$$
\lim _{t \rightarrow \infty} \mu(f(x+y)-f(x)-f(y), t \varphi(x, y))=1
$$

and

$$
\lim _{t \rightarrow \infty} v(f(x+y)-f(x)-f(y), t \varphi(x, y))=0
$$

uniformly in $X \times X$. Then $T(x)=(\mu, v)-\lim _{n \rightarrow \infty} \frac{f\left(2^{n} x\right)}{2^{n}}$ for each $x \in X$ exists and defines an additive mapping $T: X \rightarrow$ $Y$ such that if for some $\delta>0, \alpha>0$ and all $x, y \in X$,

$$
\mu(f(x+y)-f(x)-f(y), \delta \varphi(x, y))>\alpha
$$

and

$$
v(f(x+y)-f(x)-f(y), \delta \varphi(x, y))<1-\alpha,
$$

then

$$
\mu\left(T(x)-f(x), \frac{\delta}{2} \tilde{\varphi}(x, x)\right)>\alpha
$$

and

$$
v\left(T(x)-f(x), \frac{\delta}{2} \tilde{\varphi}(x, x)\right)<1-\alpha .
$$

Proof. Given $\varepsilon>0$. By (2.1.2), we can find some $t_{\circ}>0$ such that

$$
\mu(f(x+y)-f(x)-f(y), t \varphi(x, y)) \geq 1-\varepsilon
$$

and

$$
v(f(x+y)-f(x)-f(y), t \varphi(x, y)) \leq \varepsilon
$$

for all $x, y \in X$ and all $t \geq t_{\circ}$. By induction on $n$, we shall show that

$$
\mu\left(f\left(2^{n} x\right)-2^{n} f(x), t \sum_{k=0}^{n-1} 2^{n-k-1} \varphi\left(2^{k} x, 2^{k} x\right)\right) \geq 1-\varepsilon
$$

and

$$
v\left(f\left(2^{n} x\right)-2^{n} f(x), t \sum_{k=0}^{n-1} 2^{n-k-1} \varphi\left(2^{k} x, 2^{k} x\right)\right) \leq \varepsilon,
$$

for all $x \in X, t \geq t_{\circ}$ and all positive integers $n$. Putting $y=x$ in (2.1.4), we get (2.1.5) for $n=1$. Let (2.1.5) hold for some positive integer $n$. Then

$$
\mu\left(f\left(2^{n+1} x\right)-2^{n+1} f(x), t \sum_{k=0}^{n} 2^{n-k} \varphi\left(2^{k} x, 2^{k} x\right)\right)
$$




$$
\begin{gathered}
\geq \mu\left(f\left(2^{n+1} x\right)-2 f\left(2^{n} x\right), t_{\circ} \varphi\left(2^{n} x, 2^{n} x\right)\right) * \\
\mu\left(2 f\left(2^{n} x\right)-2^{n+1} f(x), t_{\circ} \sum_{k=0}^{n-1} 2^{n-k} \varphi\left(2^{k} x, 2^{k} x\right)\right) \\
\geq(1-\varepsilon) *(1-\varepsilon)=1-\varepsilon
\end{gathered}
$$

and

$$
\begin{gathered}
v\left(f\left(2^{n+1} x\right)-2^{n+1} f(x), t \sum_{k=0}^{n} 2^{n-k} \varphi\left(2^{k} x, 2^{k} x\right)\right) \\
\leq v\left(f\left(2^{n+1} x\right)-2 f\left(2^{n} x\right), t_{\circ} \varphi\left(2^{n} x, 2^{n} x\right)\right) \diamond \\
v\left(2 f\left(2^{n} x\right)-2^{n+1} f(x), t_{\circ} \sum_{k=0}^{n-1} 2^{n-k} \varphi\left(2^{k} x, 2^{k} x\right)\right)
\end{gathered}
$$

$\leq \varepsilon \diamond \varepsilon=\varepsilon$.

This completes the induction argument. Let $t=t_{\circ}$ and put $n=p$ then by replacing $x$ with $2^{n} x$ in (2.1.5), we obtain

$$
\begin{gathered}
\mu\left(\frac{f\left(2^{n+p} x\right)}{2^{n+p}}-\frac{f\left(2^{n} x\right)}{2^{n}}, \frac{t_{\circ}}{2^{n+p}} \sum_{k=0}^{p-1} 2^{p-k-1} \varphi\left(2^{n+k} x, 2^{n+k} x\right)\right) \\
\geq 1-\varepsilon
\end{gathered}
$$

and

$$
\begin{gathered}
v\left(\frac{f\left(2^{n+p} x\right)}{2^{n+p}}-\frac{f\left(2^{n} x\right)}{2^{n}}, \frac{t_{\circ}}{2^{n+p}} \sum_{k=0}^{p-1} 2^{p-k-1} \varphi\left(2^{n+k} x, 2^{n+k} x\right)\right) \\
\leq \varepsilon,
\end{gathered}
$$

for all integers $n \geq 0$ and $p>0$. The convergence of (2.1.1) and

$$
\sum_{k=0}^{p-1} 2^{-n-k-1} \varphi\left(2^{n+k} x, 2^{n+k} x\right)=\frac{1}{2} \sum_{k=n}^{n+p-1} 2^{-k} \varphi\left(2^{k} x, 2^{k} x\right)
$$

imply that for given $\delta>0$ there is $n_{\circ} \in \mathbb{N}$ such that

$$
\frac{t_{\circ}}{2} \sum_{k=n}^{n+p-1} 2^{-k} \varphi\left(2^{k} x, 2^{k} x\right)<\delta
$$

for all $n \geq n_{\circ}$ and all $p>0$. Now we deduce that from (2.1.6) that

$$
\begin{gathered}
\mu\left(\frac{f\left(2^{n+p} x\right)}{2^{n+p}}-\frac{f\left(2^{n} x\right)}{2^{n}}, \delta\right) \geq \\
\left.\mu\left(\frac{f\left(2^{n+p} x\right)}{2^{n+p}}\right)-\frac{f\left(2^{n} x\right)}{2^{n}}, \frac{t_{\circ}}{2^{n+p}} \sum_{k=0}^{p-1} 2^{p-k-1} \varphi\left(2^{n+k} x, 2^{n+k} x\right)\right) \\
\geq 1-\varepsilon
\end{gathered}
$$

and

$$
v\left(\frac{f\left(2^{n+p} x\right)}{2^{n+p}}-\frac{f\left(2^{n} x\right)}{2^{n}}, \delta\right)
$$

$$
\begin{gathered}
\left.\leq v\left(\frac{f\left(2^{n+p} x\right)}{2^{n+p}}\right)-\frac{f\left(2^{n} x\right)}{2^{n}}, \frac{t_{\circ}}{2^{n+p}} \sum_{k=0}^{p-1} 2^{p-k-1} \varphi\left(2^{n+k} x, 2^{n+k} x\right)\right) \\
\leq \varepsilon
\end{gathered}
$$

for all $n \geq n_{\circ}$ and all $p>0$. Hence $\left(\frac{f\left(2^{n} x\right)}{2^{n}}\right)$ is a Cauchy sequence in $Y$. Since $Y$ is an intuitionistic fuzzy Banach space, $\left(\frac{f\left(2^{n} x\right)}{2^{n}}\right)$ converges to some $T(x) \in Y$. Hence, we can define a mapping $T: X \rightarrow Y$ such that $T(x)=(\mu, v)-\lim _{n \rightarrow \infty} \frac{f\left(2^{n} x\right)}{2^{n}}$, namely, for each $t>0$, and $x \in X$,

$\mu\left(T(x)-\frac{f\left(2^{n} x\right)}{2^{n}}, t\right)=1$ and $v\left(T(x)-\frac{f\left(2^{n} x\right)}{2^{n}}, t\right)=0$.

Now, let $x, y \in X$. Choose any fix value of $t>0$, and $\varepsilon \in$ $(0,1)$. Since $\lim _{n \rightarrow \infty} 2^{-n} \varphi\left(2^{n} x, 2^{n} y\right)=0$, there exists $n_{1}>$ $n_{\circ}$ such that $t_{\circ} \varphi\left(2^{n} x, 2^{n} y\right)<\frac{2^{n} t}{4}$ for all $n \geq n_{1}$. Hence for each $n \geq n_{1}$, we have

$$
\begin{gathered}
\mu(T(x+y)-T(x)-T(y), t) \\
\geq \mu\left(T(x+y)-\frac{f\left(2^{n}(x+y)\right)}{2^{n}}, \frac{t}{4}\right) * \\
\mu\left(T(x)-\frac{f\left(2^{n}(x)\right)}{2^{n}}, \frac{t}{4}\right) * \\
\mu\left(T(y)-\frac{f\left(2^{n}(y)\right)}{2^{n}}, \frac{t}{4}\right) * \\
\mu\left(f\left(2^{n}(x+y)\right)-f\left(2^{n} x\right)-f\left(2^{n} y\right), \frac{2^{n} t}{4}\right)
\end{gathered}
$$

and also

$$
\begin{gathered}
\mu\left(f\left(2^{n}(x+y)\right)-f\left(2^{n} x\right)-f\left(2^{n} y\right), 2^{n} t / 4\right) \\
\geq \mu\left(f\left(2^{n}(x+y)\right)-f\left(2^{n} x\right)-f\left(2^{n} y\right), t_{\circ} \varphi\left(2^{n} x, 2^{n} y\right)\right) .
\end{gathered}
$$

Letting $n \rightarrow \infty$ in (2.1.7) and using (2.1.4), (2.1.8), we get

$$
\mu(T(x+y)-T(x)-T(y), t) \geq 1-\varepsilon
$$

for all $t>0$ and $\varepsilon \in(0,1)$. Similarly, we obtain $v(T(x+y)-T(x)-T(y), t) \leq \varepsilon$ for all $t>0$ and $\varepsilon \in(0,1)$. It follows that

$$
\mu(T(x+y)-T(x)-T(y), t)=1
$$

and

$$
v(T(x+y)-T(x)-T(y), t)=0,
$$

for all $t>0$. Therefore $T(x+y)=T(x)+T(y)$.

Lastly, suppose that for some positive $\delta$ and $\alpha$, (2.1.3) holds and

$$
\varphi_{n}(x, y)=\sum_{k=0}^{n-1} 2^{-k-1} \varphi\left(2^{k} x, 2^{k} y\right)
$$

for all $x, y \in X$. By a similar argument as in the beginning of the proof one can deduce from (2.1.3) 
$\mu\left(f\left(2^{n} x\right)-2^{n} f(x), \delta \sum_{k=0}^{n-1} 2^{n-k-1} \varphi\left(2^{k} x, 2^{k} x\right)\right) \geq \alpha$

and

$$
v\left(f\left(2^{n} x\right)-2^{n} f(x), \delta \sum_{k=0}^{n-1} 2^{n-k-1} \varphi\left(2^{k} x, 2^{k} x\right)\right) \leq 1-\alpha,
$$

for all positive integers $n$. For $s>0$, we have

$$
\begin{gathered}
\mu\left(f(x)-T(x), \delta \varphi_{n}(x, x)+s\right) \\
\geq \mu\left(f(x)-\frac{f\left(2^{n} x\right)}{2^{n}}, \delta \varphi_{n}(x, x)\right) * \\
\mu\left(\frac{f\left(2^{n} x\right)}{2^{n}}-T(x), s\right)
\end{gathered}
$$

and

$$
\begin{gathered}
v\left(f(x)-T(x), \delta \varphi_{n}(x, x)+s\right) \\
\leq v\left(f(x)-\frac{f\left(2^{n} x\right)}{2^{n}}, \delta \varphi_{n}(x, x)\right) \diamond \\
v\left(\frac{f\left(2^{n} x\right)}{2^{n}}-T(x), s\right)
\end{gathered}
$$

Combining (2.1.9), (2.1.10) and using the fact that

$$
\lim _{n \rightarrow \infty} \mu\left(\frac{f\left(2^{n} x\right)}{2^{n}}-T(x), s\right)=1
$$

and

$$
\lim _{n \rightarrow \infty} v\left(\frac{f\left(2^{n} x\right)}{2^{n}}-T(x), s\right)=0
$$

we obtain

$$
\mu\left(f(x)-T(x), \delta \varphi_{n}(x, x)+s\right) \geq \alpha
$$

and

$$
v\left(f(x)-T(x), \delta \varphi_{n}(x, x)+s\right) \leq 1-\alpha,
$$

for sufficiently large $n$. From the (upper semi) continuity of real functions $\mu(f(x)-T(x),$.$) and v(f(x)-T(x),$.$) ,$ we see that

$$
\mu\left(f(x)-T(x), \frac{\delta}{2} \tilde{\varphi}(x, x)+s\right) \geq \alpha
$$

and

$$
v\left(f(x)-T(x), \frac{\delta}{2} \tilde{\varphi}(x, x)+s\right) \leq 1-\alpha .
$$

Taking the limit $s \rightarrow \infty$, we get

$$
\mu\left(f(x)-T(x), \frac{\delta}{2} \tilde{\varphi}(x, x)\right) \geq \alpha
$$

and

$$
v\left(f(x)-T(x), \frac{\delta}{2} \tilde{\varphi}(x, x)\right) \leq 1-\alpha .
$$

Theorem 2.2. Let $X$ be a linear space and $(Y, \mu, v)$ be an intuitionistic fuzzy Banach space. Let $\varphi: X \times X \rightarrow[0, \infty)$ be a control function satisfying (2.1.1). Let $f: X \rightarrow Y$ be an uniformly approximately additive function with respect to $\varphi$. Then there is a unique additive mapping $T: X \rightarrow Y$ such that

$$
\lim _{n \rightarrow \infty} \mu(f(x)-T(x), t \tilde{\varphi}(x, x))=1
$$

and

$$
\lim _{n \rightarrow \infty} v(f(x)-T(x), t \tilde{\varphi}(x, x))=0
$$

uniformly in $X$.

Proof. The existence of uniform limit (2.2.1) immediately follows from Theorem 2.1. It remains to prove the uniqueness assertion. Let $S$ be another additive mapping satisfying (2.2.1). Choose any fix value of $c>0$. Given $\varepsilon>0$, there is some $t_{\circ}>0$ such that (2.2.1) for $T$ and $S$

$$
\begin{aligned}
\mu\left(f(x)-T(x), \frac{t}{2} \tilde{\varphi}(x, x)\right) & \geq 1-\varepsilon, \mu\left(f(x)-S(x), \frac{t}{2} \tilde{\varphi}(x, x)\right) \\
\geq & 1-\varepsilon \text { and } \\
v\left(f(x)-T(x), \frac{t}{2} \tilde{\varphi}(x, x)\right) & \leq \varepsilon, \quad v\left(f(x)-S(x), \frac{t}{2} \tilde{\varphi}(x, x)\right) \leq \varepsilon
\end{aligned}
$$

for all $x \in X$ and all $t \geq t_{\circ}$. For some $x \in X$, we can find some integer $n_{\circ}$ such that

$$
t_{\circ} \sum_{k=n}^{\infty} 2^{-k} \varphi\left(2^{k} x, 2^{k} x\right)<\frac{c}{2},
$$

for all $n \geq n_{\circ}$. Since

$$
\sum_{k=n}^{\infty} 2^{-k} \varphi\left(2^{k} x, 2^{k} x\right)
$$

$=$

$$
\begin{gathered}
\frac{1}{2^{n}} \sum_{k=n}^{\infty} 2^{-(k-n)} \varphi\left(2^{(k-n)}\left(2^{n} x\right), 2^{(k-n)}\left(2^{n} x\right)\right) \\
=\frac{1}{2^{n}} \sum_{m=0}^{\infty} 2^{-m} \varphi\left(2^{m}\left(2^{n} x\right), 2^{m}\left(2^{n} x\right)\right)=\frac{1}{2^{n}} \tilde{\varphi}\left(2^{n} x, 2^{n} x\right),
\end{gathered}
$$

we have

$$
\mu(S(x)-T(x), c) \geq \mu\left(\frac{f\left(2^{n} x\right)}{2^{n}}-T(x), c / 2\right) *
$$

$$
\mu\left(S(x)-\frac{f\left(2^{n} x\right)}{2^{n}}, c / 2\right)
$$

$$
\begin{gathered}
=\mu\left(f\left(2^{n} x\right)-T\left(2^{n} x\right), 2^{n-1} c\right) * \mu\left(S\left(2^{n} x\right)-f\left(2^{n} x\right), 2^{n-1} c\right) \\
\geq \mu\left(f\left(2^{n} x\right)-T\left(2^{n} x\right), 2^{n} t_{\circ} \sum_{k=n}^{\infty} 2^{-k} \varphi\left(2^{k} x, 2^{k} x\right)\right) * \\
\mu\left(S\left(2^{n} x\right)-f\left(2^{n} x\right), 2^{n} t_{\circ} \sum_{k=n}^{\infty} 2^{-k} \varphi\left(2^{k} x, 2^{k} x\right)\right)
\end{gathered}
$$




$$
\begin{gathered}
=\mu\left(f\left(2^{n} x\right)-T\left(2^{n} x\right), t_{\circ} \tilde{\varphi}\left(2^{n} x, 2^{n} x\right)\right) * \\
\mu\left(S\left(2^{n} x\right)-f\left(2^{n} x\right), t_{\circ} \tilde{\varphi}\left(2^{n} x, 2^{n} x\right)\right) \geq 1-\varepsilon
\end{gathered}
$$

and similarly

$$
\begin{gathered}
v(S(x)-T(x), c) \leq v\left(\frac{f\left(2^{n} x\right)}{2^{n}}-T(x), c / 2\right) \diamond \\
v\left(S(x)-\frac{f\left(2^{n} x\right)}{2^{n}}, c / 2\right) \\
\leq v\left(f\left(2^{n} x\right)-T\left(2^{n} x\right), 2^{n} t_{\circ} \sum_{k=n}^{\infty} 2^{-k} \varphi\left(2^{k} x, 2^{k} x\right)\right) \diamond \\
v\left(S\left(2^{n} x\right)-f\left(2^{n} x\right), 2^{n} t_{\circ} \sum_{k=n}^{\infty} 2^{-k} \varphi\left(2^{k} x, 2^{k} x\right)\right) \\
=v\left(f\left(2^{n} x\right)-T\left(2^{n} x\right), t_{\circ} \tilde{\varphi}\left(2^{n} x, 2^{n} x\right)\right) \diamond \\
v\left(S\left(2^{n} x\right)-f\left(2^{n} x\right), t_{\circ} \tilde{\varphi}\left(2^{n} x, 2^{n} x\right)\right) \leq \varepsilon .
\end{gathered}
$$

It follows that

$$
\mu(S(x)-T(x), c)=1 \text { and } v(S(x)-T(x), c)=0
$$

for all $c>0$. Hence $T(x)=S(x)$ for all $x \in X$.

In the next result, we consider the control function $\varphi(x, y)=\theta\left(\|x\|^{q}+\|y\|^{q}\right)$ for some $\theta>0$.

Corollary 2.3. Let $X$ be a normed linear space and $(Y, \mu, v)$ be an intuitionistic fuzzy Banach space. Let $f: X \rightarrow Y$ be a function such that for all $\theta \geq 0,0 \leq q \leq 1$

$$
\lim _{t \rightarrow \infty} \mu\left(f(x+y)-f(x)-f(y), t \theta\left(\|x\|^{q}+\|y\|^{q}\right)\right)=1
$$

and

$$
\lim _{t \rightarrow \infty} v\left(f(x+y)-f(x)-f(y), t \theta\left(\|x\|^{q}+\|y\|^{q}\right)\right)=0,
$$

uniformly in $X \times X$. Then there exists a unique additive mapping $T: X \rightarrow Y$ such that

$$
\lim _{t \rightarrow \infty} \mu\left(T(x)-f(x), \frac{2 \theta t\|x\|^{q}}{1-2^{q-1}}\right)=1
$$

and

$$
\lim _{t \rightarrow \infty} v\left(T(x)-f(x), \frac{2 \theta t\|x\|^{q}}{1-2^{q-1}}\right)=0,
$$

uniformly in $X$.

Example 2.4. Let $X$ be a Banach space and $\alpha$ and $\beta$ be real numbers. Write

$$
f(x)=\alpha x+\beta\|x\|^{q} x_{\circ}
$$

and

$$
\varphi(x, y)=\|x\|^{q}+\|y\|^{q},
$$

for all $x_{\circ}, x, y \in X$ and $0 \leq q \leq 1$. Then

$$
\tilde{\varphi}(x, y) \sum_{n=0}^{\infty} 2^{-n} \varphi\left(2^{n} x, 2^{n} y\right)=\frac{2^{1-q}\left(\|x\|^{q}+\|y\|^{q}\right)}{2^{1-q}-1},
$$

for all $x, y \in X$. For each intuitionistic fuzzy norm $(\mu, v)$, we have

$$
\begin{gathered}
\mu(f(x+y)-f(x)-f(y), t \varphi(x, y)) \\
=\mu\left(\beta x_{\circ}\left(\|x+y\|^{q}-\|x\|^{q}-\|y\|^{q}\right),\left(\|x\|^{q}+\|y\|^{q}\right) t\right)
\end{gathered}
$$

and

$$
\begin{gathered}
v(f(x+y)-f(x)-f(y), t \varphi(x, y)) \\
=v\left(\beta x_{\circ}\left(\|x+y\|^{q}-\|x\|^{q}-\|y\|^{q}\right),\left(\|x\|^{q}+\|y\|^{q}\right) t\right),
\end{gathered}
$$

for all $x, y \in X$ and $t \in \mathbb{R}$. Thus

$$
\mu(f(x+y)-f(x)-f(y), t \varphi(x, y)) \geq \mu\left(\beta x_{\circ}, t / 2\right)
$$

and

$$
v(f(x+y)-f(x)-f(y), t \varphi(x, y)) \leq v\left(\beta x_{\circ}, t / 2\right),
$$

for all $x, y \in X$ and $t \in \mathbb{R}$. Hence

$$
\lim _{t \rightarrow \infty} \mu(f(x+y)-f(x)-f(y), t \varphi(x, y))=1
$$

and

$$
\lim _{t \rightarrow \infty} v(f(x+y)-f(x)-f(y), t \varphi(x, y))=0
$$

uniformly in $X \times X$. Therefore the condition of Corollary 2.3 are fulfilled.

Now, we are giving our second intuitionistic fuzzy Hyers-Ulam-Rassias type theorem (non-uniform version).

Theorem 2.5. Let $X$ be a linear space and $\left(Z, \mu^{\prime}, v^{\prime}\right)$ be an intuitionistic fuzzy normed space. Let $\psi: X \times X \rightarrow Z$ be a function such that for some $0<\alpha<2$,

$$
\mu^{\prime}(\psi(2 x, 2 y), t) \geq \mu^{\prime}(\alpha \psi(x, y), t)
$$

and

$$
v^{\prime}(\psi(2 x, 2 y), t) \leq v^{\prime}(\alpha \psi(x, y), t),
$$

for all $x, y \in X$ and $t>0$. Let $(Y, \mu, v)$ be an intuitionistic fuzzy Banach space and let $f: X \rightarrow Y$ be a $\psi$-approximately additive mapping in the sense that

$$
\mu(f(x+y)-f(x)-f(y), t) \geq \mu^{\prime}(\psi(x, y), t)
$$

and

$$
v(f(x+y)-f(x)-f(y), t) \leq v^{\prime}(\psi(x, y), t),
$$

for all $x, y \in X$ and $t>0$. Then there exists a unique additive mapping $T: X \rightarrow Y$ such that

$$
\mu(f(x)-T(x), t) \geq \mu^{\prime}\left(\frac{2 \psi(x, x)}{2-\alpha}, t\right)
$$


and

$$
v(f(x)-T(x), t) \leq v^{\prime}\left(\frac{2 \psi(x, x)}{2-\alpha}, t\right)
$$

for all $x \in X$ and $t>0$.

Proof. Put $y=x$ in (2.5.2), we get

$$
\mu(f(2 x)-2 f(x), t) \geq \mu^{\prime}(\psi(x, x), t)
$$

and

$$
v(f(2 x)-2 f(x), t) \leq v^{\prime}(\psi(x, x), t),
$$

for all $x \in X$ and $t>0$. Using (2.5.1) and induction on $n$, we obtain

$$
\mu^{\prime}\left(\psi\left(2^{n} x, 2^{n} x\right), t\right) \geq \mu^{\prime}\left(\alpha^{n} \psi(x, x), t\right)
$$

and

$$
v^{\prime}\left(\psi\left(2^{n} x, 2^{n} x\right), t\right) \leq v^{\prime}\left(\alpha^{n} \psi(x, x), t\right),
$$

for all $x \in X$ and $t>0$. Replacing $x$ by $2^{n-1} x$ in (2.5.3) and using (2.5.4), we get

$$
\left.\begin{array}{l}
\mu\left(f\left(2^{n} x\right)-2 f\left(2^{n-1} x\right), t\right) \geq \mu^{\prime}\left(\alpha^{n-1} \psi(x, x), t\right) \text { and } \\
v\left(f\left(2^{n} x\right)-2 f\left(2^{n-1} x\right), t\right) \leq v^{\prime}\left(\alpha^{n-1} \psi(x, x), t\right) .
\end{array}\right\}
$$

It follows that

$$
\mu\left(\frac{f\left(2^{n} x\right)}{2^{n}}-\frac{f\left(2^{n-1} x\right)}{2^{n-1}}, \frac{t}{2^{n}}\right) \geq \mu^{\prime}\left(\frac{1}{\alpha} \psi(x, x), \frac{t}{\alpha^{n}}\right)
$$

and

$$
v\left(\frac{f\left(2^{n} x\right)}{2^{n}}-\frac{f\left(2^{n-1} x\right)}{2^{n-1}}, \frac{t}{2^{n}}\right) \leq v^{\prime}\left(\frac{1}{\alpha} \psi(x, x), \frac{t}{\alpha^{n}}\right) .
$$

Thus

$$
\mu\left(\frac{f\left(2^{n} x\right)}{2^{n}}-\frac{f\left(2^{n-1} x\right)}{2^{n-1}},\left(\frac{\alpha}{2}\right)^{n} t\right) \geq \mu^{\prime}\left(\frac{1}{\alpha} \psi(x, x), t\right)
$$

and

$$
v\left(\frac{f\left(2^{n} x\right)}{2^{n}}-\frac{f\left(2^{n-1} x\right)}{2^{n-1}},\left(\frac{\alpha}{2}\right)^{n} t\right) \leq v^{\prime}\left(\frac{1}{\alpha} \psi(x, x), t\right)
$$

for all $x \in X, t>0$ and $n \geq 1$. Therefore

$$
\begin{gathered}
\mu\left(\frac{f\left(2^{n} x\right)}{2^{n}}-\frac{f\left(2^{m} x\right)}{2^{m}}, \sum_{k=m+1}^{n}\left(\frac{\alpha}{2}\right)^{k} t\right) \\
=\mu\left(\sum_{k=m+1}^{n}\left(\frac{f\left(2^{k} x\right)}{2^{k}}-\frac{f\left(2^{k-1} x\right)}{2^{k-1}}\right), \sum_{k=m+1}^{n}\left(\frac{\alpha}{2}\right)^{k} t\right) \\
\geq \mu^{\prime}\left(\frac{1}{\alpha} \psi(x, x), t\right)
\end{gathered}
$$

and

$$
\begin{gathered}
v\left(\frac{f\left(2^{n} x\right)}{2^{n}}-\frac{f\left(2^{m} x\right)}{2^{m}}, \sum_{k=m+1}^{n}\left(\frac{\alpha}{2}\right)^{k} t\right) \\
=v\left(\sum_{k=m+1}^{n}\left(\frac{f\left(2^{k} x\right)}{2^{k}}-\frac{f\left(2^{k-1} x\right)}{2^{k-1}}\right), \sum_{k=m+1}^{n}\left(\frac{\alpha}{2}\right)^{k} t\right) \\
\leq v^{\prime}\left(\frac{1}{\alpha} \psi(x, x), t\right)
\end{gathered}
$$

for all $x \in X, t>0$ and $n>m \geq 0$. Hence

$$
\mu\left(\frac{f\left(2^{n} x\right)}{2^{n}}-\frac{f\left(2^{m} x\right)}{2^{m}}, t\right) \geq \mu^{\prime}\left(\frac{1}{\alpha} \psi(x, x), \frac{t}{\sum_{k=m+1}^{n}\left(\frac{\alpha}{2}\right)^{k}}\right)
$$

and

$$
v\left(\frac{f\left(2^{n} x\right)}{2^{n}}-\frac{f\left(2^{m} x\right)}{2^{m}}, t\right) \leq v^{\prime}\left(\frac{1}{\alpha} \psi(x, x), \frac{t}{\sum_{k=m+1}^{n}\left(\frac{\alpha}{2}\right)^{k}}\right),
$$

for all $x \in X, t>0$ and $n>m \geq 0$. Since $\lim _{s \rightarrow \infty} \mu^{\prime}\left(\frac{1}{\alpha} \psi(x, x), s\right) \quad=\quad 1 \quad$ and $\lim _{s \rightarrow \infty} \nu^{\prime}\left(\frac{1}{\alpha} \psi(x, x), s\right)=0$; also $\sum_{n=0}^{\infty}\left(\frac{\alpha}{2}\right)^{n}<\infty$. This shows that $\left(\frac{f\left(2^{n} x\right)}{2^{n}}\right)$ is a Cauchy sequence in the intuitionistic fuzzy Banach space $(Y, \mu, v)$, therefore it is convergent to some $T(x) \in Y$. So we can define a mapping $T: X \rightarrow Y$ by $T(x)=(\mu, v)-\lim _{n \rightarrow \infty} \frac{f\left(2^{n} x\right)}{2^{n}}$. For $x, y \in X$ and $t>0$, it follows from (2.5.2) that

$\mu\left(f\left(2^{n}(x+y)\right)-f\left(2^{n} x\right)-f\left(2^{n} y\right), t\right) \geq \mu^{\prime}\left(\psi\left(2^{n} x, 2^{n} y\right), t\right)$

and similarly

$$
\geq \mu^{\prime}\left(\alpha^{n} \psi(x, y), t\right) \geq \mu^{\prime}\left(\psi(x, y),\left(\frac{1}{\alpha}\right)^{n} t\right)
$$

$v\left(f\left(2^{n}(x+y)\right)-f\left(2^{n} x\right)-f\left(2^{n} y\right), t\right) \leq v^{\prime}\left(\psi\left(2^{n} x, 2^{n} y\right), t\right)$

$$
\leq v^{\prime}\left(\psi(x, y),\left(\frac{1}{\alpha}\right)^{n} t\right)
$$

Thus

$$
\begin{gathered}
\mu\left(\frac{f\left(2^{n}(x+y)\right)}{2^{n}}-\frac{f\left(2^{n} x\right)}{2^{n}}-\frac{f\left(2^{n} y\right)}{2^{n}}, t\right) \\
\geq \mu^{\prime}\left(\psi(x, y),\left(\frac{2}{\alpha}\right)^{n} t\right)
\end{gathered}
$$

and

$$
\begin{gathered}
v\left(\frac{f\left(2^{n}(x+y)\right)}{2^{n}}-\frac{f\left(2^{n} x\right)}{2^{n}}-\frac{f\left(2^{n} y\right)}{2^{n}}, t\right) \\
\leq v^{\prime}\left(\psi(x, y),\left(\frac{2}{\alpha}\right)^{n} t\right)
\end{gathered}
$$

for all $n$. Furthermore,

$$
\mu(T(x+y)-T(x)-T(y), t)
$$




$$
\begin{gathered}
\geq \mu\left(T(x+y)-\frac{f\left(2^{n}(x+y)\right)}{2^{n}}, \frac{t}{4}\right) * \\
\mu\left(T(x)-\frac{f\left(2^{n} x\right)}{2^{n}}, \frac{t}{4}\right) * \mu\left(T(y)-\frac{f\left(2^{n} y\right)}{2^{n}}, \frac{t}{4}\right) * \\
\mu\left(\frac{f\left(2^{n}(x+y)\right)}{2^{n}}-\frac{f\left(2^{n} x\right)}{2^{n}}-\frac{f\left(2^{n} y\right)}{2^{n}}, \frac{t}{4}\right)
\end{gathered}
$$

and

$$
\begin{gathered}
v(T(x+y)-T(x)-T(y), t) \\
\leq v\left(T(x+y)-\frac{f\left(2^{n}(x+y)\right)}{2^{n}}, \frac{t}{4}\right) \diamond \\
v\left(T(x)-\frac{f\left(2^{n} x\right)}{2^{n}}, \frac{t}{4}\right) \diamond v\left(T(y)-\frac{f\left(2^{n} y\right)}{2^{n}}, \frac{t}{4}\right) \\
\diamond v\left(\frac{f\left(2^{n}(x+y)\right)}{2^{n}}-\frac{f\left(2^{n} x\right)}{2^{n}}-\frac{f\left(2^{n} y\right)}{2^{n}}, \frac{t}{4}\right)
\end{gathered}
$$

Letting $n \rightarrow \infty$ in (2.5.7) and (2.5.8), we get

$$
\mu(T(x+y)-T(x)-T(y), t)=1
$$

and

$$
v(T(x+y)-T(x)-T(y), t)=0,
$$

for all $x, y \in X$ and $t>0$. Thus $T(x+y)=T(x)+T(y)$. This means that $T$ satisfies the Cauchy equation and so it is additive. Using (2.5.6) with $m=0$, and for all $x \in X$ and $t>0$, we get

$$
\begin{gathered}
\mu(T(x)-f(x), t) \geq \mu\left(T(x)-\frac{f\left(2^{n} x\right)}{2^{n}}, \frac{t}{2}\right) * \\
\mu\left(\frac{f\left(2^{n} x\right)}{2^{n}}-f(x), \frac{t}{2}\right) \\
\geq \mu\left(T(x)-\frac{f\left(2^{n} x\right)}{2^{n}}, \frac{t}{2}\right) * \mu^{\prime}\left(\frac{1}{\alpha} \psi(x, x), \frac{t}{2 \sum_{k=1}^{n}\left(\frac{\alpha}{2}\right)^{k}}\right)
\end{gathered}
$$

and

$$
\begin{gathered}
v(T(x)-f(x), t) \leq v\left(T(x)-\frac{f\left(2^{n} x\right)}{2^{n}}, \frac{t}{2}\right) \diamond \\
v\left(\frac{f\left(2^{n} x\right)}{2^{n}}-f(x), \frac{t}{2}\right) \leq v\left(T(x)-\frac{f\left(2^{n} x\right)}{2^{n}}, \frac{t}{2}\right) \\
\diamond v^{\prime}\left(\frac{1}{\alpha} \psi(x, x), \frac{t}{2 \sum_{k=1}^{n}\left(\frac{\alpha}{2}\right)^{k}}\right)
\end{gathered}
$$

Letting $n \rightarrow \infty$ in (2.5.9), we get

$$
\begin{gathered}
\mu(T(x)-f(x), t) \geq \mu^{\prime}\left(\frac{2 \psi(x, x)}{\alpha}, \frac{t}{2 \sum_{k=1}^{\infty}\left(\frac{\alpha}{2}\right)^{k}}\right) \\
=\mu^{\prime}\left(\frac{2 \psi(x, x)}{2-\alpha}, t\right)
\end{gathered}
$$

and

$$
v(T(x)-f(x), t) \leq v^{\prime}\left(\frac{2 \psi(x, x)}{\alpha}, \frac{t}{2 \sum_{k=1}^{\infty}\left(\frac{\alpha}{2}\right)^{k}}\right)
$$

$$
=v^{\prime}\left(\frac{2 \psi(x, x)}{2-\alpha}, t\right)
$$

for all $x \in X$ and $t>0$. The uniqueness of $T$ can be proved on the same lines as in Theorem 2.2.

Example 2.6. Let $X$ be a normed space. Suppose that $(\mu, v)$ and $\left(\mu^{\prime}, v^{\prime}\right)$ be intuitionistic fuzzy norms on $X$ and $\mathbb{R}$ respectively, defined in Example 1.4 and $\varphi:(0, \infty) \rightarrow(0, \infty)$ be a function such that $\varphi(2 r)<\alpha \varphi(r)$ for all $r>0$ and $\alpha \in(0,2)$. Define

$$
\psi(x, y)=\varphi(\|x\|)+\varphi(\|y\|)+\varphi(\|x+y\|)
$$

for each $x, y \in X$. Let $x_{\circ} \in X$ be a unit vector. Define $f$ : $X \rightarrow X$ by $f(x)=x+\varphi(\|x\|) x_{\circ}$. Then for each $x, y \in X$ and $t>0$, we have

$$
\begin{gathered}
\mu(f(x+y)-f(x)-f(y), t) \\
=\frac{t}{t+|\varphi(\|x\|)+\varphi(\|y\|)+\varphi(\|x+y\|)| \cdot\left\|x_{\circ}\right\|} \\
\geq \frac{t}{t+|\varphi(\|x\|)+\varphi(\|y\|)+\varphi(\|x+y\|)|}=\mu^{\prime}(\psi(x, y), t)
\end{gathered}
$$

and similarly,

$$
\begin{gathered}
v(f(x+y)-f(x)-f(y), t) \\
=\frac{t}{t+|\varphi(\|x\|)+\varphi(\|y\|)+\varphi(\|x+y\|)| .\left\|x_{\circ}\right\|} \leq v^{\prime}(\psi(x, y), t) .
\end{gathered}
$$
Furthermore,

$\mu^{\prime}(\psi(2 x, 2 y), t)=\frac{t}{t+\psi(2 x, 2 y)} \geq \frac{t}{t+\alpha \psi(x, y)}=\mu^{\prime}(\psi(x, y), t)$ and

$v^{\prime}(\psi(2 x, 2 y), t)=\frac{t}{t+\psi(2 x, 2 y)} \leq \frac{t}{t+\alpha \psi(x, y)}=v^{\prime}(\psi(x, y), t)$, for all $x, y \in X$ and $t>0$. Therefore, by Theorem 2.5, there exists a unique additive mapping $T: X \rightarrow Y$ such that for each $x \in X$ and $t>0$

$$
\begin{aligned}
\mu(f(x)-T(x), t) \geq & \mu^{\prime}\left(\frac{2 \psi(x, x)}{2-\alpha}, t\right) \text { and } v(f(x)-T(x), t) \\
& \leq v^{\prime}\left(\frac{2 \psi(x, x)}{2-\alpha}, t\right)
\end{aligned}
$$

\section{Acknowledgment}

The authors gratefully acknowledge the financial support from King Abdulaziz University, Jeddah, Saudi Arabia.

\section{References}

[1] A. S. Al-Fhaid and S. A. Mohiuddine, On the Ulam stability of mixed type QA mappings in IFN-spaces, Adv. Difference Equ., 2013, (2013).

[2] A. Alotaibi and S. A. Mohiuddine, On the stability of a cubic functional equation in random 2-normed spaces, Adv. Difference Equ., 2012, (2012). 
[3] T. Aoki, On the stability of the linear transformation in Banach spaces, J. Math. Soc. Japan, 2, 64-66 (1950).

[4] L. C. Barros, R. C. Bassanezi and P. A. Tonelli, Fuzzy modelling in population dynamics, Ecol. Model, 128, 27-33 (2000).

[5] I. -S. Chang, Higher ring derivation and intuitionistic fuzzy stability, Abstr. Appl. Analy. Article ID 503671, 16 pages (2012).

[6] A. L. Fradkov and R. J. Evans, Control of chaos: methods and applications in engineering, Chaos, Solitons \& Fractals, 29, 33-56 (2005).

[7] R. Giles, A computer program for fuzzy reasoning, Fuzzy Sets Syst., 4, 221-234 (1980).

[8] I. Goleţ, On probabilistic 2-normed spaces. Novi Sad J. Math., 35, 95-102 (2005).

[9] L. Hong and J.Q. Sun, Bifurcations of fuzzy nonlinear dynamical systems, Commun. Nonlinear Sci. Numer. Simul., 1, 1-12 (2006).

[10] D. H. Hyers, On the stability of the linear functional equation, Proc. Nat. Acad. Sci. USA, 27, 222-224 (1941).

[11] O. Kaleva and S. Seikkala, On fuzzy metric spaces, Fuzzy Sets Syst., 12, 215-229 (1984).

[12] K. -W. Jun and H. -M. Kim, On the Hyers-Ulam-Rassias stability of a general cubic functional equation, Math. Ineq. Appl., 6, 87-95 (2003).

[13] K. -W. Jun and H. -M. Kim, On the Hyers-Ulam stability of a generalzed quadratic and additive functional equation, Bull. Korean Math. Soc., 42, 133-148 (2005).

[14] Y. -S. Lee and S. -Y. Chung, Stability of the Jensen type functional equation, Banach J. Math. Anal., 1, 91-100 (2007).

[15] J. Madore, Fuzzy physics, Ann. Phys., 219, 187-198 (1992).

[16] A. K. Mirmostafaee and M.S. Moslehian, Fuzzy versions of Hyers-Ulam-Rassias theorem, Fuzzy Sets Syst., 159, 720729 (2008).

[17] S. A. Mohiuddine, Stability of Jensen functional equation in intuitionistic fuzzy normed space, Chaos, Solitons \& Fract., 42, 2989-2996 (2009).

[18] VN Vapnik, AY Chervonenkis. On the uniform convergence of relative frequencies of events to their probabilities. Theory of Probability \& Its Applications, (1971).

[19] S. A. Mohiuddine and M.A. Alghamdi, Stability of functional equation obtained through a fixed-point alternative in intuitionistic fuzzy normed spaces, Adv. Difference Equ., 2012, (2012).

[20] S. A. Mohiuddine and A. Alotaibi, Fuzzy stability of a cubic functional equation via fixed point technique, Adv. Difference Equ., 2012, (2012)

[21] S. A. Mohiuddine, A. Alotaibi and S.M. Alsulami, Ideal convergence of double sequences in random 2-normed spaces. Adv. Difference Equ., 2012, (2012).

[22] S. A. Mohiuddine, A. Alotaibi and M. Obaid, Stability of various functional equations in non-Archimedean intuitionistic fuzzy normed spaces, Discrete Dyn. Nat. Soc. 2012, Article ID 234727, 16 pages (2012).

[23] S. A. Mohiuddine, M. Cancan and H. Şevli, Intuitionistic fuzzy stability of a Jensen functional equation via fixed point technique, Math. Comput. Modelling, 54, 2403-2409 (2011).

[24] S. A. Mohiuddine and Q.M.D. Lohani, On generalized statistical convergence in intuitionistic fuzzy normed space, Chaos, Solitons \& Fract., 42, 1731-1737 (2009).
[25] S.A. Mohiuddine and H. Şevli, Stability of Pexiderized quadratic functional equation in intuitionistic fuzzy normed space, J. Comput. Appl. Math., 235, 2137-2146 (2011).

[26] M. Mursaleen, On statistical convergence in random 2normed spaces. Acta Sci. Math. (Szeged), 76, 101-109 (2010).

[27] H. D. Mathur and H. V. Manjunath. Frequency stabilization using fuzzy logic based controller for multi-area power system. The South Pacific Journal of Natural Science, 25, 2229 (2008).

[28] M. Mursaleen and S. A. Mohiuddine, Statistical convergence of double sequences in intuitionistic fuzzy normed spaces, Chaos, Solitons Fract., 41, 2414-2421 (2009).

[29] M. Mursaleen and S. A. Mohiuddine, Nonlinear operators between intuitionistic fuzzy normed spaces and Fréchet differentiation, Chaos, Solitons Fract., 42, 1010-1015 (2009).

[30] M. Mursaleen and S. A. Mohiuddine, On stability of a cubic functional equation in intuitionistic fuzzy normed spaces, Chaos, Solitons Fract., 42, 2997-3005 (2009).

[31] M. Mursaleen and S. A. Mohiuddine, On lacunary statistical convergence with respect to the intuitionistic fuzzy normed space, J. Comput. Appl. Math., 233, 142-149 (2009).

[32] M. Mursaleen, V. Karakaya and S.A. Mohiuddine, Schauder basis, separability, and approximation property in intuitionistic fuzzy normed space, Abstract and Applied Analysis 2010, Article ID 131868, 14 pages (2010).

[33] A. Najati, Hyers-Ulam-Rassias stability of a cubic functional equation, Bull. Korean Math. Soc., 44, 825-840 (2007).

[34] C. Park, J. R. Lee, D. Y. Shin, Generalized Ulam-Hyers stability of random homomorphisms in random normed algebras associated with the Cauchy functional equation, Appl. Math. Lett., 25, 200-205 (2012).

[35] T. M. Rassias, On the stability of functional equations and a problem of Ulam, Acta. Appl. Math., 62, 123-130 (2000).

[36] R. Saadati and J. H. Park, On the intuitionistic fuzzy topological spaces. Chaos, Solitons \& Fractals, 27, 331-344 (2006).

[37] S. M. Ulam, Problems in Modern Mathematics. Science ed., John Wiley \& Sons: New York; (Chapter VI, Some Questions in Analysis: Section 1, Stability), (1940).

[38] T. Z. Xu, J. M. Rassias and W.X. Xu, Intuitionistic fuzzy stability of a general mixed additive cubic equation, J. Math. Physics, 51, 063519 (2010).

[39] Y. Yilmaz, On some basic properties of differentiation in intuitionistic fuzzy normed spaces, Math. Comput. Model., 52, 448-458 (2010).

[40] L.A. Zadeh, Fuzzy sets, Inform Control, 8, 338-353 (1965). 


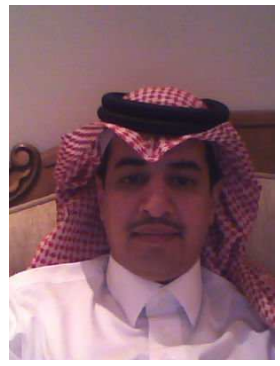

Abdullah Alotaibi is
full Professor in Mathematics
at King Abdulaziz University,
Jeddah, Saudi Arabia
and presently the chairman of
the department. He received
his M.Sc. from University
of Missouri-Columbia,
Columbia, Missouri,
U.S.A and Ph. D. Form the

University of Nottingham, UK. He has published about 50 research papers in various journals of high repute. His main research interests are in the field of Complex Analysis, Summability Theory, Approximation Theory and Fixed Point Theory.

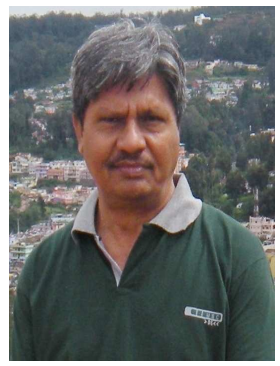

M. Mursaleen is a full Professor in Mathematics at Aligarh Muslim University, Aligarh. He has has written 05 book chapters and published about 190 research papers in various journals of international repute. He has visited a number of countries including USA \& UK and gave about 32 talks there and had joint research work with faculty members of the host institutions. He is member of the Editorial Board of various scientific journals and served as a member of various international scientific and organizing bodies. He is reviewer for Mathematical Reviews (USA) and many scientific journals. His main research interests are Sequence Spaces, Summability Theory, Approximation Theory, Fuzzy Mathematics and Measures of Noncompactness.

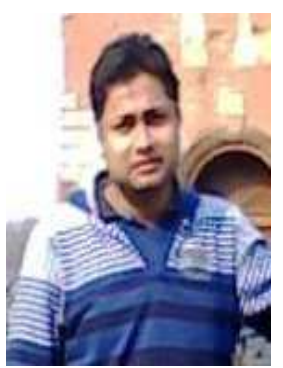

Hemen Dutta is a faculty member in the department of Mathematics of Gauhati University, India. He did his M.Phil and Ph.D in the field of Analysis. His research interests are in the areas of Functional Analysis and Fuzzy Mathematics. He has published research articles in reputed international journals is referee and editor of mathematical journals.

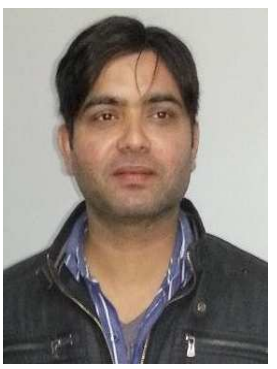

from National Board for

Higher Mathematics (NBHM), Department of Atomic Energy, Government of India. His main research interests are in the field of Sequences Spaces, Measures of Noncompactness, Fixed Point Theory, Approximation Theory, Summability Theory and Fixed Point Theory. He has published more than seventy research papers in well reputed national and international journals and two book chapters. He is reviewer for Mathematical Reviews (USA) and referee for many scientific journals. He is also member of the editorial board of some mathematical journals. 